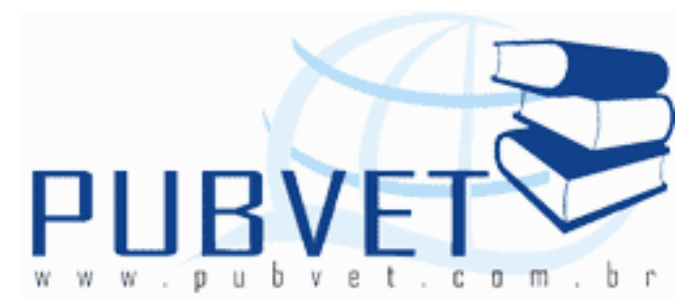

PUBVET, Publicações em Medicina Veterinária e Zootecnia.

Disponível em: <https://doi.org/10.31533/pubvet.v02n11a456>.

\title{
Diagnóstico ultra-sonográfico de fratura craniana e contusão cerebral em cão - relato de caso
}

Bernardo Fernandes Lopes ${ }^{1}$, Vicente Colombi Silva ${ }^{1}$, Adrienne Bretas Lanis ${ }^{1}$, Daniel Capucho de Oliveira ${ }^{2}$, Fabiano Séllos Costa ${ }^{3}$

1-Médico Veterinário autônomo, CLIMEV- DIAGNOPET, Vitória, ES.

2- Acadêmico do curso de medicina veterinária do Centro de Ciências Agrárias da Universidade Federal do Espírito Santo - ES.

3- Professor Adjunto de Diagnóstico por Imagem e Clínica Médica de Pequenos Animais - Centro de Ciências Agrárias da Universidade Federal do Espírito Santo - ES.

\section{RESUMO:}

Relata-se no presente trabalho um caso de traumatismo craniano em um cão de três meses de idade, onde o exame ultra-sonográfico intracraniano permitiu o diagnóstico de fratura em osso parietal direito com conseqüente contusão cerebral. Conclui-se que este método de exame pode ser útil para avaliação de traumatismos cranianos, particularmente em animais que ainda apresentem a fontanela aberta.

Palavras chave: Fratura craniana, ultra-som, cão. 
Lopes, B.F., Silva, V.C., Lanis, A.B. et al. Diagnóstico ultra-sonográfico de fratura craniana e contusão cerebral em cão - relato de caso. PUBVET, Londrina, V. 2, N. 47, Art\#456, Nov4, 2008.

\section{Ultrasonographic diagnosis of cranial fracture and cerebral contusion in a dog - case report}

\section{ABSTRACT:}

It is reported in this paper a case of cranial traumatism in a three months aged dog, in which the intracranial ultrasonographic examination allowed the diagnosis of right parietal bone fracture with consequent cerebral contusion. It follows that this examination method can be useful for cranial traumatisms evaluation, particularly in animals that still have the fontanelle opened.

Keywords: Cranial traumatism, ultrasound, dogs

\section{INTRODUÇAO E REVISÃO DE LITERATURA:}

Traumatismos cranianos em cães são de ocorrência freqüente na rotina clinica de animais de companhia. Como método simplificado de classificação de lesões cerebrais intra-axiais, é citado o edema cerebral, contusão e hematoma cerebral, lesões axonal difusa e do tronco cerebral e lesão vascular. As lesões cerebrais também podem ser divididas de acordo com a localização, sendo que as lesões no interior do parênquima são denominadas intra-axiais e aquelas que afetam as estruturas ao redor, como as meninges ou o liqüor, são denominadas de extra-axiais (Farrow, 2006).

O exame radiográfico do crânio é por muitas vezes realizado na rotina veterinária para diagnóstico de lesões cranianas, entretanto, este método semiológico apresenta limitações. Informações importantes podem ser obtidas referentes às estruturas ósseas cranianas, mas uma avaliação dos tecidos moles não é possível por meio do exame radiográfico (ANDRADE NETO, 2004; CARVALHO, et al., 2007.

O diagnóstico por imagem de lesões cerebrais decorrentes de traumas atualmente são realizados na maioria dos casos por tomografia 
Lopes, B.F., Silva, V.C., Lanis, A.B. et al. Diagnóstico ultra-sonográfico de fratura craniana e contusão cerebral em cão - relato de caso. PUBVET, Londrina, V. 2, N. 47, Art\#456, Nov4, 2008.

computadorizada (TC) e ressonância magnética (RM), sendo pouco freqüente o uso da ultra-sonografia intracraniana (FARROW, 2006).

Segundo Carvalho et al. (2007), a ultra-sonografia transcraniana é uma alternativa viável para a auxiliar a rotina clínica de cães e gatos, podendo ser útil na detecção de alterações estruturais e morfológicas do cérebro. Segundo Hudson et al. (1991), a maioria dos exames na medicina veterinária é realizado em neonatos por permitirem uma melhor janela acústica.

Dentre as indicações clínicas para realização de ultra-sonografia craniana, podemos citar hemorragias, isquemias, edema, hidrocefalia, cistos, abscessos, ventriculites, malformações e massas tumorais. Em muitos casos a acurácia no diagnóstico pode ser considerada superior à tomografia computadorizada, apresentando vantagens de ter menor custo e por não ser uma técnica invasiva (CARVALHO \& ANDRADE NETO, 2004).

\section{RELATO DE CASO:}

Relata-se no presente trabalho o caso de um cão da raça Lhasa Apso, com um mês de idade, atendido emergencialmente no Hospital Veterinário CLIMEV - ES, apresentando-se com histórico de trauma recente na região do crânio originado pelo membro de outro cão que convivia no mesmo ambiente.

Ao exame clínico observou-se a inspeção que o animal apresentava-se com incoordenação, ataxia, vocalização, estrabismo canto-lateral e crises convulsivas seqüenciais. Após intervenção inicial, o animal foi encaminhado para exame ultra-sonográfico da região encefálica utilizando-se a como janela acústica a região da fontanela.

A avaliação ultra-sonográfica intracraniana revelou a presença de fragmento ósseo em osso parietal projetando-se para o encéfalo (Figura 1). Adicionalmente observou-se uma imagem hiperecogênica de contornos 
Lopes, B.F., Silva, V.C., Lanis, A.B. et al. Diagnóstico ultra-sonográfico de fratura craniana e contusão cerebral em cão - relato de caso. PUBVET, Londrina, V. 2, N. 47, Art\#456, Nov4, 2008.

pobremente definidos, mensurando aproximadamente $1,2 \mathrm{~cm}$ em maior diâmetro e distorcendo ventrículo lateral direito (Figura 2). Com base nestes achados foi possível estabelecer o diagnóstico de fratura craniana em osso parietal direito, promovendo uma contusão encefálica com formação de hematoma e edema adjacente à lesão.

Posteriormente ao exame foi dada continuidade à terapia para o quadro neurológico com corticóide de ação ultra-rápida, furosemida, diazepam e fluidoterapia de suporte. O animal apresentou melhora progressiva, havendo remissão parcial dos sinais neurológicos após sete dias de internação, sendo posteriormente encaminhado para continuidade do tratamento em casa. Após 30 dias foi observada ao exame clínico uma total remissão dos sinais neurológicos observados ao momento do exame ultra-sonográfico.

\section{DISCUSSÃO E CONCLUSÕES:}

Concordando com os relatos encontrados na literatura (ANDRADE NETO, 2004; CARVALHO, et al., 2007), o exame ultra-sonográfico neste caso foi útil para o diagnóstico das lesões encefálicas decorrente do trauma craniano e permitiu um melhor direcionamento da terapia a ser instituída.

O traumatismo craniano deve ser considerado como uma emergência clínica e necessita de uma rápida intervenção por parte do médico veterinário. Um preciso diagnóstico das alterações intracranianas é necessário para o estabelecimento de uma adequada conduta terapêutica e para o estabelecimento do prognóstico. Apesar de Farrow (2006) citar que os exames de tomografia computadorizada e ressonância magnética são determinantes para o diagnóstico de lesões intracranianas em cães e gatos, estes métodos de diagnóstico por imagem muitas vezes são difíceis de serem realizados em nosso país. Desta forma a ultra-sonografia encefálica pode ser uma alternativa viável, principalmente para cães jovens que sofrem trauma craniano. 
Lopes, B.F., Silva, V.C., Lanis, A.B. et al. Diagnóstico ultra-sonográfico de fratura craniana e contusão cerebral em cão - relato de caso. PUBVET, Londrina, V. 2, N. 47, Art\#456, Nov4, 2008.

Assim como relatado por Hudson et al. (1991), a imagem intracraniana foi possível de ser formada por intermédio da fontanela que ainda estava presente. Desta forma, o fato do animal do presente relato ter apenas 30 dias favoreceu a realização do exame.

De acordo com as citações de Farrow, (2006), os achados ultrasonográficos permitiram classificar o trauma craniano neste caso como primário, de golpe e intra-axial.

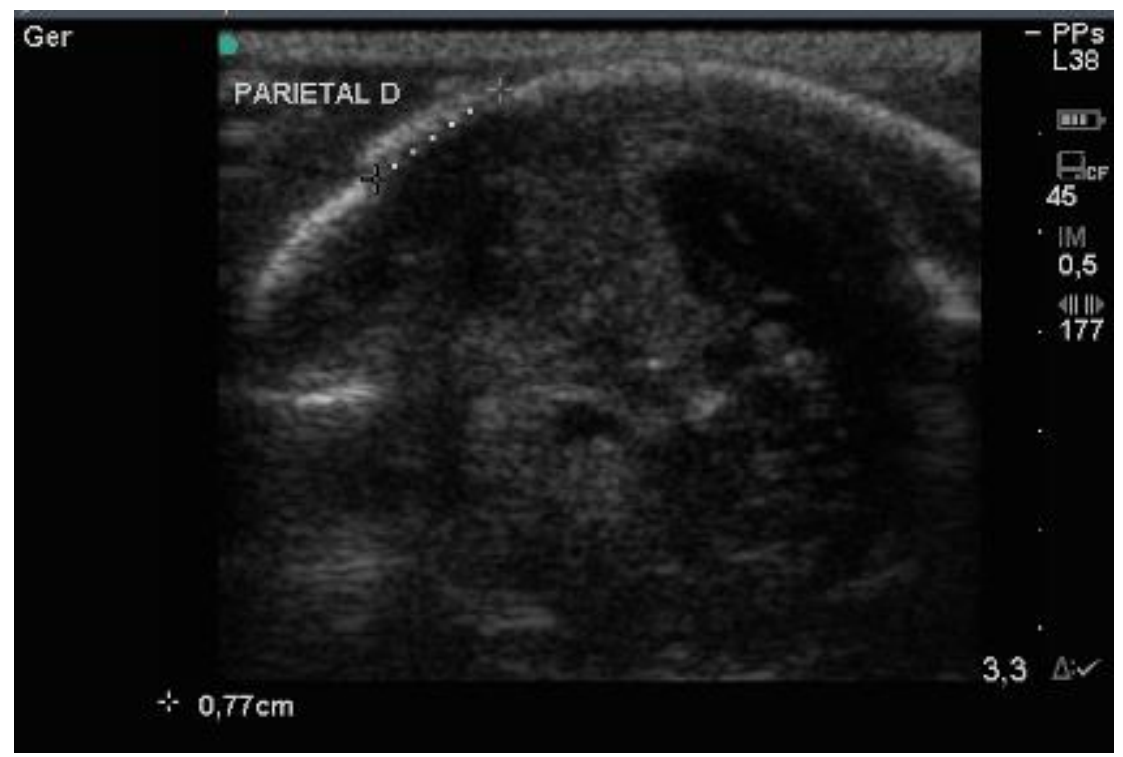

Figura 1 - Ultra-sonografia intracraniana em cão após traumatismo craniano demonstrando fragmento ósseo em osso parietal projetando-se para o encéfalo. 
Lopes, B.F., Silva, V.C., Lanis, A.B. et al. Diagnóstico ultra-sonográfico de fratura craniana e contusão cerebral em cão - relato de caso. PUBVET, Londrina, V. 2, N. 47, Art\#456, Nov4, 2008.

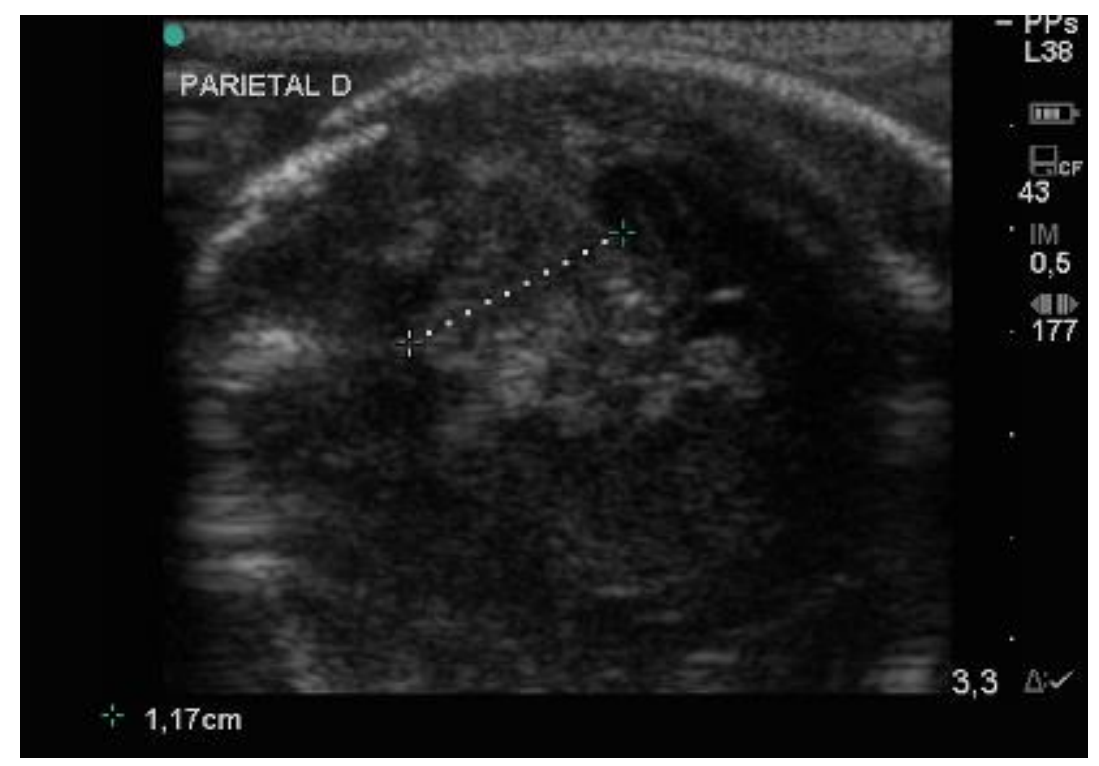

Figura 2 - Ultra-sonografia intracraniana em cão após traumatismo craniano demonstrando imagem hiperecogênica e com limites irregulares compatíveis com

\section{REFERÊNCIAS:}

CARVALHO, C.F.; ANDRADE NETO Ecoencefalografia. In: Ultra-sonografia em Pequenos Animais, CARVALHO, C.F. (ed.), São Paulo: Roca, 2004, p.265-277.

CARVALHO, C.F.; ANDRADE NETO, J.P.; JIMENEZ, C.D. ; DINIZ, S.A.; CERRI, G.G.; CHAMMAS, M.E.C. Ultra-sonografia transcraniana em cães com distúrbios neurológicos de origem central. Arquivo Brasileiro de Medicina Veterinária e Zootecnia, v.59, n.6, 2007.

FARROW, C.S. Doenças e lesões cerebrais. In: FARROW, C.S. (ed). Veterinária Diagnóstica do Cão e Gato. São Paulo : Roca, 2005, p.220-237.

HUDSON, J.A.; SIMPSON, S.T.; COX, N.R. et al. Ultrasonographic examination of the normal canine neonatal brain. Veterinary Radiolology and Ultrasound.., v.32, p.50-59, 1991. 